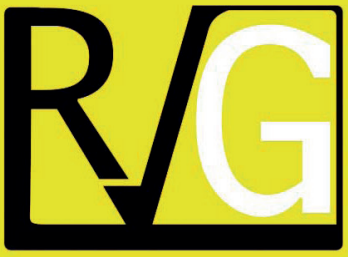

Julio - Septiembre, 2021

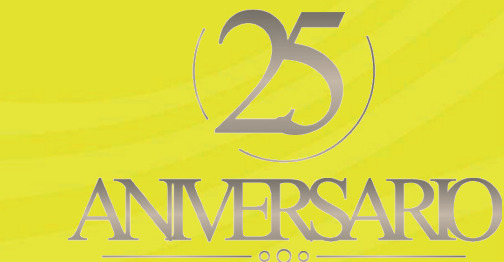

ANMERSARO
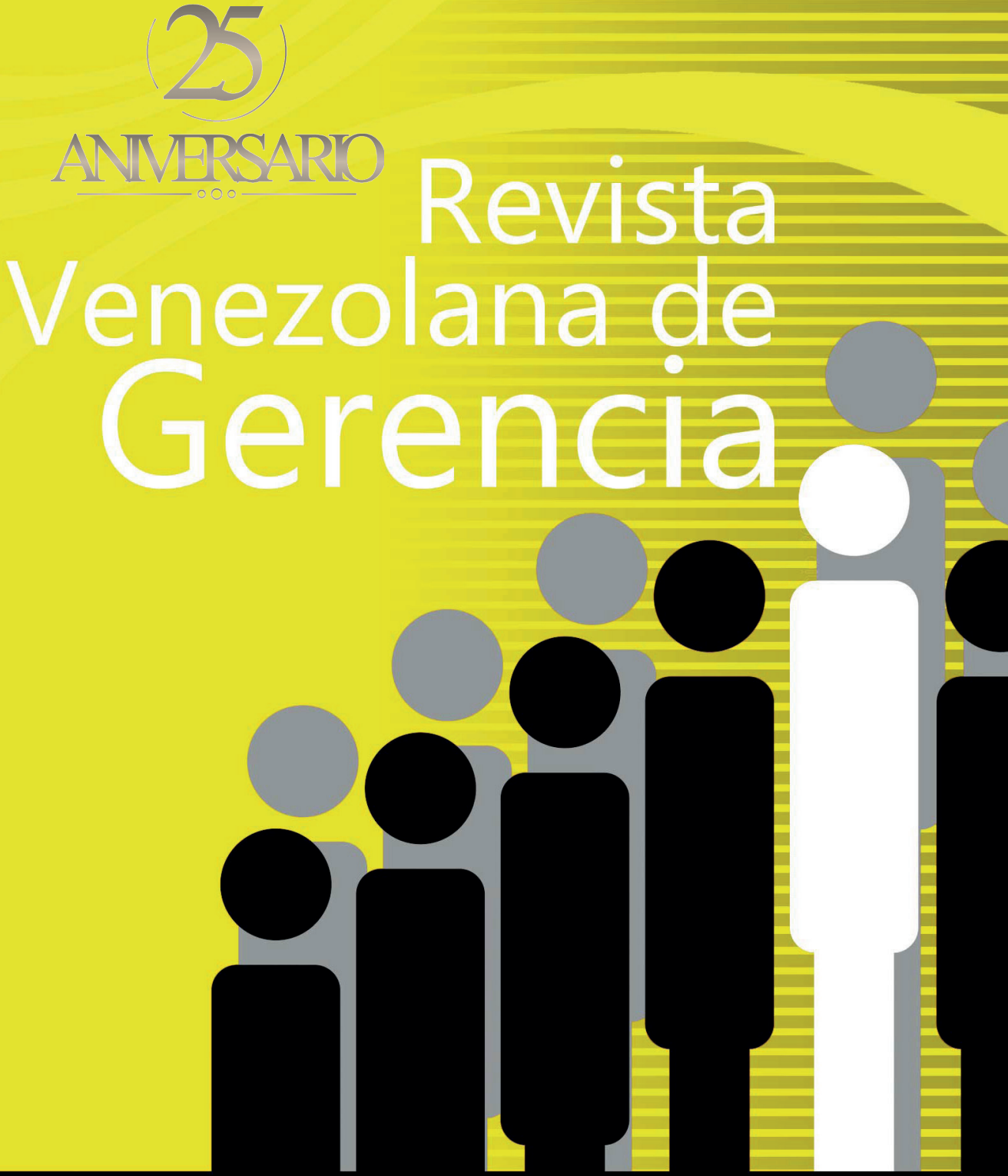

UNIVERSIDAD DEL ZULIA (LUZ)

Facultad de Ciencias Económicas y Sociales Centro de Estudios de la Empresa

ISSN 1315-99

Esta obra está bajo una licencia de Creative Comm Reconocimiento-NoComercial-Compartirlgual 3.0 Unpo http://creativecommons.org/licenses/by-nc-sa/3.0/deed.es 
COMO CITAR: Ríos Lara, G. F., Lara, R. E., Medina Guerrero, W. E., Romero Arévalo, D. A. (2021) Posicionamiento estratégico de la Federación Deportivade Tungurahua, Ecuador. Revista Venezolana de Gerencia, 26(95), 962-977. https://doi. org/10.52080/rvgluz.27.95.31
Universidad del Zulia (LUZ)

Revista Venezolana de Gerencia (RVG)

Año 26 No. 95 Julio-Septiembre 2021, 962-977

ISSN 1315-9984 / e-ISSN 2477-9423

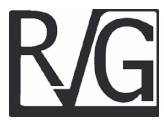

\title{
Posicionamiento estratégico de la Federación Deportivade Tungurahua, Ecuador
}

\author{
Ríos Lara, Geovanny Fabricio* \\ León Lara, Roxana Elizabeth* \\ Medina Guerrero, Wilson Eduardo** \\ Romero Arévalo, Dennis Adolfo ${ }^{* \star * *}$
}

\section{Resumen}

El posicionamiento representa una estrategia que ha ido adquiriendo cada vez más relevancia para las diferentes organizaciones. Esta situación se ha visto reflejada en la mayor utilización del posicionamiento como una herramienta indispensable para competir en un ambiente ampliamente globalizado. Por esta razón, el presente estudio se centra en diseñar estrategias de posicionamiento para la Federación Deportiva de Tungurahua, las cuales permitirán, como establece la literatura, obtener una mayor participación de mercado y por ende mayor rentabilidad. Metodológicamente tiene un diseño descriptivo-no experimenta, con aplicación de entrevista estructuradas a personal clave de la Federación Deportiva de Tungurahua, así como el uso de las matrices para el diagnóstico estratégico planteadas por David (2004). El principal resultado, fue la ausencia de un plan estratégico que ayude en la gestión de la organización, generando con ello un estancamiento empresarial y la consecuente pérdida de mercado. Se concluye sobre la necesidad de generar estrategias de posicionamiento buscando satisfacer las necesidades presentes y futuras de los distintos consumidores a través de un plan estratégico de marketing, las cuales se proponen.

Palabras clave: estrategias; posicionamiento estratégico; marketing; participación de mercado.

\section{Recibido: $25.02 .21 \quad$ Aceptado: 16.05 .21}

* Magíster Ejecutivo en Dirección de Empresas con Énfasis en Gerencia estratégica; Universidad Regional Autónoma de los Andes - Ecuador; Docente; Universidad Técnica de Ambato - Ecuador; geovannyfrios@ uta.edu.ec, https://orcid.org/0000-0001-6162-8086

** Magíster en Gestión del Talento Humano; Universidad Técnica de Ambato - Ecuador; Docente; Instituto Superior Tecnológico Tena - Ecuador; rleon@institutos.gob.ec, https://orcid.org/0000-0001-8887-3081

*** Magíster en Administración Financiera y Comercio Internacional; Universidad Técnica de Ambato - Ecuador; Docente; Instituto España - Ecuador; edumg1438@hotmail.com, https://orcid.org/0000-0002-1252-3189

**** Magíster en Administración de Empresas Mención Planeación; Pontificia Universidad Católica - Ecuador; Director Financiero; Mancomunidad de Cotopaxi - Ecuador; dennisromerito@hotmail.it, https://orcid. org/0000-0002-7116-3750 


\title{
Strategic positioning of the Sports Federation of Tungurahua, Ecuador
}

\begin{abstract}
Positioning represents a strategy that has become increasingly relevant for different organizations. This situation has been reflected in the greater use of positioning as an indispensable tool to compete in a widely globalized environment. For this reason, this study focuses on designing positioning strategies for the Tungurahua Sports Federation, which will allow, as established in the literature, to obtain a greater market share and therefore greater profitability. Methodologically, it has a descriptive design - no experimentation, with application of structured interviews to key personnel of the Sports Federation of Tungurahua, as well as the use of matrices for strategic diagnosis proposed by David (2004). The main result was the absence of a strategic plan to help in the management of the organization, thereby generating business stagnation and the consequent loss of the market. It concludes on the need to generate positioning strategies seeking to satisfy the present and future needs of different consumers through a strategic marketing plan, which are proposed.
\end{abstract}

Keywords: strategies; Strategic positioning; marketing; market share

\section{Introducción}

Las empresas luchan por diferenciarse en el sector donde compiten, dado que los productos y servicios se han vuelto cada vez más comunes, por lo que, no es suficiente que una compañía se posicione. Por lo mismo, desean integrar con éxito los servicios y los productos, al posicionar la marca en torno a las capacidades empresariales y los objetivos de los clientes (Carpio et al., 2019). Señalan Suarez et al (2021:265), que "un aspecto fundamental de la planificación estratégica es sin duda la consideración de la competencia como factor determinante en la configuración de las estrategias organizacionales".

El posicionar una marca es uno de los elementos fundamentales del marketing, tanto para productos de consumo como para servicios, es la única forma de una marca de proporcionar valor a sus clientes, creando una imagen del producto o servicio en la mente de un cliente objetivo (Panamá et al., 2019). En ese sentido, el posicionamiento define cómo la oferta de la marca es única, cómo proporciona un beneficio distintivo a los clientes. Por ello, las empresas utilizan el marketing para comunicar su posición en el mercado e influir en la percepción de los productos o servicios de la marca, en relación con las alternativas disponibles de los competidores (Panamá et al., 2019). En ese sentido, una estrategia de localización exitosa se basa en una comprensión profunda del mercado en el que desea competir, se lo direcciona para identificar en qué se diferencia la empresa de los competidores y las condiciones y oportunidades en el mercado. 
Un gran error que cometen muchas empresas es asumir que el posicionamiento es solo una estrategia de marketing, lo cual, debe ser uno de los pilares de la estrategia empresarial (López et al., 2018). Después de todo, no se puede posicionar un producto o servicio como una oferta de alta calidad en su marketing si el producto o servicio en sí no puede respaldar esas afirmaciones (Franco, Restrepo y Sánchez 2014). Las organizaciones deben definirlo claramente a lo largo de la cadena de valor; de lo contrario, la comunicación pierde el enfoque y puede volverse confusa.

En el caso de las organizaciones deportivas, al igual que el resto de las organizaciones, aún de distinta naturaleza, se conciben con una estructura que oriente su funcionamiento al alcance de los objetivos que persiga, $y$ al igual que otras organizaciones requiere de una gestión que incluya el marketing como vía para insertarse en el mercado en el cual desarrolla sus actividades.

En el Ecuador, la gestión de las federaciones deportivas se encuentra a cargo del Ministerio del Deporte, del cual recibe lineamientos así como una partida presupuestaria para su funcionamiento. Un elemento a destacar con relación a estas federaciones, es que las estrategias de marketing empleadas hasta el momento han condicionado la penetración de las mismas hacia diferentes segmentos del mercado, es decir se observan decisiones que no parecen obedecer al mayor interés de las federaciones. Sobre ello, apuntan Jeanpert \& Paché (2016), al aseverar que la inestabilidad organizacional de orden administrativo, ha creado desconfianza en la población debido a que sus decisiones son variantes y de sesgo político.

Dentro del contexto de estudio, la Federación Deportiva de Tungurahua constituye una institución que contribuye al desarrollo del deporte de la provincia, sin embrago en los últimos años, ha venido perdiendo paulatinamente sus cliente y en consecuencia su posición en el mercado, los deportistas (clientes) buscan otras alternativas para poder entrenarse, situación que se encuentra asociada a factores como ausencia de estrategias de posicionamiento y escasa publicidad, entre los más destacados.

En cuanto a la ausencia de estrategias de posicionamiento, ha ocasionado que exista un estancamiento empresarial y la pérdida de mercado (Romero y Moreno, 2018), dado que la competencia sigue creciendo, y hoy en día son muchos los lugares que ofrecen entrenamientos permanentes en diferentes disciplinas, lo cual hace que el deportista opte por estos nuevos lugares.

Adicional a lo señalado, la situación se complica por la escaza publicidad que la federación emplea, impidiendo de esta manera que más personas conozcan los servicios que la institución oferta al público en general (Fuchs \& Diamantopoulos, 2010; Hume \& Hume, 2015); siendo este aspecto una debilidad a ser considerada para mejorar la situación futura de la institución.

Con estos antecedentes, el objetivo del estudio se centra en el diseño de estrategias que permitan posicionar en el mercado a la Federación Deportiva de Tungurahua a través de un diagnóstico previo estableciendo fortalezas, debilidades, oportunidades y amenazas que permita conocer la situación estratégica actual de la institución y proyectar con ello acciones que ayuden a mejorar su desempeño y 
posicionamiento en el mercado.

En ese sentido, el abordaje metodológico tiene un diseño de tipo descriptivo-no experimental (Hernández, Fernández y Baptista, 2014). Con relación a la modalidad de investigación se hizo una revisión bibliográfica y de campo; en primera instancia, se procesó información recabada en fuentes secundarias; es decir, investigaciones realizadas con anterioridad, libros, revistas científicas, entre otros documentos.

Por otro lado, el levantamiento de información implicó a la población conformada por aquellas personas directamente vinculadas con los aspectos administrativos y de marketing, para conocer la realidad de la institución, tanto desde el punto de vista interno (fortalezas y debilidades) como desde el punto de vista de los factores exógenos (oportunidades y amenazas) relacionados con el posicionamiento en el mercado. Para ello, se diseñaron dos entrevistas estructuradas, la primera aplicada al administrador de la Federación Deportiva de Tungurahua. Mientras que, la segunda entrevista se aplicó al responsable de las relaciones públicas de la institución.

Con la información recabada a través de las entrevistas y con el soporte teórico resultado de la revisión bibliográfica, se procedió a elaborar el diagnóstico situacional de la federación, empleando las matrices planteadas por David (2003): Evaluación de Factores Internos (MEFI) y la Matriz de Evaluación de Factores Externos (MEFE). Las salidas de éstas permitieron alimentar la tercera y última denominada Matriz FODA, cuyos resultados analizados a la luz de las necesidades de la federación permitieron generar una serie de estrategias encaminadas a mejorar el posicionamiento de la organización en el mercado.

\section{Posicionamiento estratégico: consideraciones esenciales}

Cuando una empresa persigue una orientación proactiva de mercado, buscará identificar y responder a las necesidades latentes y no satisfechas de los clientes en el mercado. Para identificar las necesidades insatisfechas, la empresa requerirá una mentalidad de aprendizaje exploratoria impulsada por el mercado. En consecuencia, la empresa probablemente considerará estrategias de posicionamiento que coincidan con la mentalidad de la empresa y las competencias relacionadas.

El posicionamiento estratégico surgió en el plan estructural del marketing durante los años sesenta y principios de la década de los años ochenta (Zaheer y Rashid, 2017). Su evolución epistemológica evolucionó de las discusiones de segmentación del mercado y público objetivo. Dentro del entorno del marketing, el posicionamiento es el acto de diseñar una oferta e imagen empresarial destinada a conseguir u ocupar un lugar distinguible en la mente del público objetivo. Para Abdullah (2015) el término posicionamiento hace referencia al sitio que el producto $u$ ofrecimiento ocupa en la mente de los consumidores con relación a sus atributos importantes que son comparados con otros ofrecimientos de la competencia. De otra manera, se establece que, el posicionamiento de marca representa el uso que hace una organización de todos los elementos que dispone para crear y mantenerse en la mente del mercado meta. 
La percepción del posicionamiento de marca está vinculada al proceso de diferenciación y no al de estandarización (Kitchen \& Burgmann, 2015). Tener el mejor posicionamiento es ser la primera persona $u$ objeto en la mente de los consumidores que generan mayor participación de mercado y rentabilidad (Copulsky \& Wolf, 1990; Nogales, 2006). A lo largo del tiempo, la flexibilidad de los programas de marketing, innovación continua y reputación, ha permitido alcanzar el liderazgo en el mercado (Chang \& Tun-Min, 2015).

Es por esto, que hablar de posicionamiento de marca es referirse a ocupar un lugar en la mente del consumidor. Dicho en otras palabras, implica que el consumidor pueda reconocer las características del producto o servicio, comparar y diferenciar con los de la competencia, de forma que, define un elemento distinto, superior y único (Abdullah, 2015; Maritz \& Du Toit, 2018).

Por su parte, Wijaya y Sirine (2016) en un nivel básico, lo posicionan de acuerdo con los atributos del producto. Sin embargo, son el nivel menos deseable del posicionamiento según el enfoque de marca. Pues, los competidores llegan a simular de manera fácil e igualitaria cada uno de ellos y, aún más importante, los clientes no son atraídos por ellos, sino por los beneficios que otorga.

La distinción es un requisito previo para la sostenibilidad en los mercados competitivos, y el posicionamiento es un proceso para crear una distinción (Bonilla et al., 2018). En este mismo orden de ideas, Rasooli et al. (2018) establecieron al posicionamiento como una manifestación clara, superior y deseable de un producto en la mente de los clientes en el mercado de destino, de acuerdo con los productos de las empresas rivales.
Para Suarez et al (2021), el nuevo panorama en el cual se desempeñan las organizaciones, las somete a un proceso de inestabilidad y exigencia de ajuste constantes, por ello es difícil prever el comportamiento de las variable que le rodean y de allí la necesidad de sustentar las decisiones en un pensamieno estratégico. Acorde con lo anterior Ruiz et al., (2018) expresan que el análisis estratégico es fundamental para identificar y comprender los factores que debe abordar una posición estratégica. La elección de la posición estratégica influye en los recursos y capacidades clave de una empresa al elegir una estrategia para competir con éxito contra rivales en una industria.

La planificación estratégica del marketing es el proceso que puede permitir a una empresa concentrar sus recursos limitados en las mayores oportunidades disponibles para aumentar las ventas y lograr una ventaja competitiva sostenible. En este sentido, un posicionamiento estratégico debe centrarse en el concepto clave de que la satisfacción del cliente es el objetivo principal.

En marketing, posicionamiento ha llegado a significar el proceso mediante el cual los especialistas en marketing intentan crear una imagen o identidad en la mente de su mercado objetivo para su producto, marca u organización. El posicionamiento de la marca es el acto de diseñar la oferta y la imagen de la empresa para que ocupe un lugar distinto y valorado en la mente del cliente objetivo. Un buen posicionamiento de marca ayuda a guiar la estrategia de marketing al aclarar de qué se trata una marca, en qué es única, en qué se parece a las marcas de la competencia y por qué los consumidores la compran.

La estrategia del posicionamiento se centra en buscar en el mundo 
productos y servicios desde el punto de vista de los clientes, y luego obtener un espacio deseable y vacante en la mente de los consumidores (Bonilla, Delgado y Fajardo, 2020). Hoy en día, la industria del deporte ha crecido y se ha generalizado, y existen numerosas marcas organizativas en la industria. Para identificar el estatus en todos los sectores de la sociedad incluidas las organizaciones de servicios, es necesario de una planificación estratégica direccionada al marketing.

Dentro de la sistematización de la estrategia como herramienta de dirección se han elaborado modelos y técnicas que han sido aplicados con la finalidad de examinar diferentes variables que posibiliten llegar a la formulación definitiva de opciones estratégicas. Sobre esta base, la aplicación de metodologías y técnicas de matrices han sido ampliamente aceptados por ser un método didáctico que permite ir por etapas mostrando información de forma gráfica del contexto interno (fortalezas y debilidades) así como también del externo (oportunidades y amenazas) (Villalón et al., 2017).

En este orden de ideas, David (2003) establece que las matrices estratégicas como la Matriz FODA, la Matriz de Evaluación de Factores Internos (MEFI) y la Matriz de Evaluación de Factores Externos (MEFE) constituyen herramientas que ayudan a identificar, evaluar y seleccionar adecuadamente las estrategias más idóneas para un determinado caso. Adicional a ello, en su teoría argumenta que para su construcción de estas dos últimas el juicio intuitivo acertado es siempre necesario para determinar los valores y calificaciones que mejor se adapten.

El proceso o sistema de gestión de marketing estratégico ha sido diseñado para ayudar a la dirección a tomar decisiones estratégicas, así como a crear visiones estratégicas. Rajiv et al. (2020) mencionan que muchos errores estratégicos ocurren como resultado de no enfatizar hasta ahora redes de colaboración fuertes en un determinado sector, lo que ha limitado a las empresas replicar estructuras adyacentes. Al respecto, Vidaller (2014) argumenta que dichas prácticas de replicación, son limitadas debido a los escasos recursos en finanzas, personal, habilidades $y$ actitud hacia el marketing.

Ahora bien, según la percepción de Herrera (2017) existe una falta de disponibilidad de expertos y especialistas en marketing dentro del sector de público deportivo. Por lo tanto, no se lleva a cabo una planificación estratégica adecuada, lo que da como resultado altas tasas de fracaso empresarial. La falta de marketing y la deficiente planificación del marketing estratégico ha sido un detonante de los fracasos organizacionales (Kitchen \& Burgmann, 2015). En este mismo contexto, Maritz \& Du Toit (2018) indican que las instituciones públicas aún carecen de la capacidad de comercializar sus productos de manera efectiva. Como lo confirma Mourad (2010), pues reveló que existe una relación positiva entre la falta de planificación estratégica de marketing y el fracaso empresarial.

\section{Diagnóstico y diseño estratégico para la Federación Deportiva de Tungurahua}

Las entrevistas efectuadas, tanto al administrador como al responsable de las relaciones públicas de la institución, permitieron recabar información relevante acerca la Federación 
Deportiva, en torno a los elementos o factores de orden interno y externo que determinan su actividad.

Esta data, contrastada con la revisión bibliográfica sobre el tema, fue la base inicial para la aplicación de las matrices propuestas por David (2003) (FODA, MEFI y MEFE), dando como resultado la elaboración de un diagnóstico estratégico tanto a nivel interno como externo a la Federación Deportiva de Tungurahua, mediante el cual se determinaron las fortalezas, debilidades, oportunidades y amenazas las mismas que son descritas a continuación (tabla 1):

\section{Tabla 1}

\section{Matriz MEFI}

\begin{tabular}{|c|c|c|c|c|}
\hline \multicolumn{2}{|r|}{ Factores Internos } & \multirow{2}{*}{$\begin{array}{c}\text { Ponderación } \\
(0-1)\end{array}$} & \multirow{2}{*}{$\begin{array}{c}\text { Clasificación } \\
(1-4)\end{array}$} & \multirow{2}{*}{$\begin{array}{l}\text { Resultado } \\
\text { Ponderado }\end{array}$} \\
\hline & & & & \\
\hline \multirow{7}{*}{ 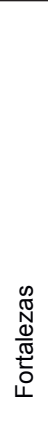 } & $\begin{array}{l}\text { Institución con años de trayectoria a nivel na- } \\
\text { cional }\end{array}$ & 0,07 & 4 & 0,28 \\
\hline & Complejos deportivos & 0,05 & 4 & 0,20 \\
\hline & $\begin{array}{l}\text { Conocimiento de directivos en el ámbito de- } \\
\text { portivo }\end{array}$ & 0,05 & 4 & 0,20 \\
\hline & $\begin{array}{l}\text { Ajedrez y tenis de campo deportes con ma- } \\
\text { yor demanda a pesar que no existe mayor } \\
\text { inversión }\end{array}$ & 0,05 & 4 & 0,20 \\
\hline & Portafolio de productos y/o servicios & 0,05 & 4 & 0,20 \\
\hline & Flexibilidad en la disponibilidad de horarios. & 0,05 & 4 & 0,20 \\
\hline & Federados (deportistas) & 0,07 & 4 & 0,28 \\
\hline \multirow{12}{*}{$\begin{array}{l}\text { D } \\
\frac{0}{0} \\
\frac{0}{0} \\
\frac{0}{\overline{0}} \\
0 \\
0\end{array}$} & Alta rotación del personal administrativo & 0,07 & 1 & 0,07 \\
\hline & $\begin{array}{l}\text { Distribución inequitativa en las actividades } \\
\text { colectivas e individuales }\end{array}$ & 0,05 & 1 & 0,05 \\
\hline & $\begin{array}{l}\text { Contratación de personal inadecuado (entre- } \\
\text { nadores) }\end{array}$ & 0,05 & 1 & 0,05 \\
\hline & Infraestructura básica & 0,07 & 1 & 0,07 \\
\hline & Falta de publicidad & 0,05 & 1 & 0,05 \\
\hline & Alto número de deserción de deportistas & 0,07 & 1 & 0,07 \\
\hline & $\begin{array}{l}\text { Falta de mantenimiento a los complejos de- } \\
\text { portivos y maquinaria }\end{array}$ & 0,05 & 1 & 0,05 \\
\hline & $\begin{array}{l}\text { Escaso compromiso del personal administra- } \\
\text { tivo y de servicios con el trabajo }\end{array}$ & 0,05 & 1 & 0,05 \\
\hline & Alta rotación de deportistas & 0,05 & 1 & 0,05 \\
\hline & Logros deportivos a escala nacional & 0,05 & 1 & 0,05 \\
\hline & $\begin{array}{l}\text { Ausencia de planes de incentivos o bonos } \\
\text { que permitan fidelizar a los clientes }\end{array}$ & 0,05 & 1 & 0,05 \\
\hline & Total & 1 & & 2,17 \\
\hline
\end{tabular}

Nota. La tabla en cuestión establece aquellos factores internos relevantes para la institución estableciendo una ponderación y calificación con la finalidad de obtener un resultado final que indique la situación actual en torno a los factores evaluados.

Fuente: elaboración propia 
El resultado obtenido luego de realizado la evaluación a través de la matriz de los factores internos (MEFI) refleja un valor de 2,17 lo que indica un resultado por debajo de la media, es decir, las debilidades son más que las fortalezas dentro de la institución. Por lo tanto, se deberían plantear estrategias que ayuden a convertir esas debilidades en fortalezas enfocándose en conseguir el posicionamiento en el mercado.

Por otro lado, la matriz de evaluación de factores externos (MEFE), una vez identificado los factores externos claves se les asigna una ponderación y calificación a cada uno de ellos, se identifica amenazas y oportunidades de mayor o menor impacto. El resultado ponderado máximo que se puede obtener es de 4. Mientras que, el resultado ponderado mínimo es de 1 . Por tanto, el valor obtenido luego de haber realizado el análisis de la matriz MEFE de la Federación Deportiva de Tungurahua, fue de 2,90. Es decir, que la institución se encuentra en una industria que afronta graves amenazas externas y es ahí donde se tienen que diseñar estrategias para contrarrestar esta situación (tabla 2)

\section{Tabla 2}

\section{Matriz MEFE}

\begin{tabular}{|c|c|c|c|c|}
\hline \multicolumn{2}{|r|}{ Factores Externos Claves } & \multirow{2}{*}{$\begin{array}{c}\text { Ponderación } \\
(0-1)\end{array}$} & \multirow{2}{*}{$\begin{array}{c}\text { Clasificación } \\
(1-4)\end{array}$} & \multirow{2}{*}{$\begin{array}{l}\text { Resultado } \\
\text { Ponderado }\end{array}$} \\
\hline & & & & \\
\hline \multirow{11}{*}{ 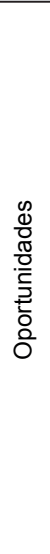 } & Ingresos económicos por auspicio privado & 0,06 & 4 & 0,24 \\
\hline & Nuevos deportistas & 0,06 & 4 & 0,24 \\
\hline & Fidelización del deportista & 0,06 & 4 & 0,24 \\
\hline & Autogestión & 0,06 & 4 & 0,24 \\
\hline & Ley del deporte & 0,06 & 4 & 0,24 \\
\hline & $\begin{array}{l}\text { Capacitación y manejo tecnológico de los empleados } \\
\text { y entrenadores }\end{array}$ & 0,06 & 4 & 0,24 \\
\hline & $\begin{array}{l}\text { Creación de proyectos que aporten al desarrollo pro- } \\
\text { fesional }\end{array}$ & 0,06 & 4 & 0,24 \\
\hline & Convenio con instituciones públicas y privadas & 0,06 & 4 & 0,24 \\
\hline & Desarrollo de proyectos & 0,06 & 4 & 0,24 \\
\hline & Fortalecimiento del marco legal y administrativo & 0,06 & 4 & 0,24 \\
\hline & Implementar nuevas formas de pago & 0,05 & 4 & 0,20 \\
\hline \multirow{6}{*}{$\begin{array}{l}\text { D } \\
\mathbb{N} \\
\mathbb{J} \\
\frac{1}{0} \\
\frac{E}{<}\end{array}$} & Escaso interés de los deportistas en disciplinas nuevas & 0,05 & 1 & 0,05 \\
\hline & $\begin{array}{l}\text { Presencia de otros centros que fomentan la actividad } \\
\text { física }\end{array}$ & 0,05 & 1 & 0,05 \\
\hline & $\begin{array}{l}\text { Diminución del presupuesto asignado por la Secretaria } \\
\text { Nacional del Deporte }\end{array}$ & 0,05 & 1 & 0,05 \\
\hline & Problemas nutricionales en los deportistas & 0,05 & 1 & 0,05 \\
\hline & Problemas extradeportivos de los deportistas & 0,05 & 1 & 0,05 \\
\hline & Total & 1 & & 2,90 \\
\hline
\end{tabular}

Nota. La tabla en cuestión establece aquellos factores externos relevantes para la institución, estableciendo una ponderación y calificación con la finalidad de obtener un resultado final que indique la situación actual en torno a los factores evaluados.

Fuente: elaboración propia 


\section{Cuadro 1}

\section{Matriz FODA Cruzada - Federación Deportiva de Tungurahua}

\begin{tabular}{ll}
\hline \multicolumn{1}{c}{ Fortalezas } & \multicolumn{1}{c}{ Debilidades } \\
\hline $\begin{array}{l}\text { F1. Institución con años de trayecto- } \\
\text { ria a nivel nacional }\end{array}$ & $\begin{array}{l}\text { D1. Alta rotación de personal administrativo por } \\
\text { temas coyunturales }\end{array}$ \\
\hline $\begin{array}{l}\text { F2. Complejos deportivos } \\
\text { D2. Distribución inequitativa en las actividades } \\
\text { colectivas e individuales }\end{array}$ \\
\hline $\begin{array}{l}\text { F3. Conocimiento de directivos en el } \\
\text { ámito deportivo }\end{array}$ & $\begin{array}{l}\text { D3. Contratación de personal inadecuado (en- } \\
\text { trenadores) }\end{array}$ \\
\hline $\begin{array}{l}\text { F4. Ajedrez y tenis de campo depor- } \\
\text { no con mayor demanda a pesar que }\end{array}$ & D4. Infraestructura básica \\
\hline $\begin{array}{l}\text { F5. Portafolio de productos y/o ser- } \\
\text { vicios }\end{array}$ & D5. Falta de publicidad \\
\hline F6. Flexibilidad en horarios & D6. Alto número de deserción de deportistas \\
\hline F7. Federados (deportistas) & $\begin{array}{l}\text { D7. Falta de mantenimiento a los complejos } \\
\text { deportivos y maquinaria }\end{array}$ \\
\hline Fo EXPLOTAR & $\begin{array}{l}\text { D8. Escaso compromiso del personal adminis- } \\
\text { trativo y de servicios con el trabajo }\end{array}$ \\
\hline $\begin{array}{l}\text { D9. Abandono de la práctica deportiva por falta } \\
\text { de recursos }\end{array}$ \\
\hline D10. Logros deportivos a escala nacional \\
\hline $\begin{array}{l}\text { D11. Ausencia de planes de incentivos o bonos } \\
\text { que permitan fidelizar a los clientes }\end{array}$ \\
\hline
\end{tabular}

01. Ingresos económicos por auspicio empresa privada

O2. Nuevos deportistas

\section{O3. Fidelización del deportista}

O4. Autogestión de la FDT

O5. Ley del deporte

O6. Capacitación y manejo tecnológico de los empleados y entrenadores

O7. Creación de proyectos que aporten al desarrollo profesional

O8. Convenio con instituciones públicas y privadas

O9. Desarrollo de proyectos

O10. Fortalecimiento del marco legal y administrativo

011. Implementar nuevas formas de pago
F1:F2:F5:08:09 Efectuar talleres y seminarios gratuitos promoviendo la realización del deporte y su afectación en la salud en las instituciones públicas y privadas que brinde apretura.

F5:O13 Implementar el pago del servicio mediante tarjetas de crédito, a fin de poder competir en el mercado ya que la competencia ya brinda este servicio y así poder llegar a un segmento más amplio de clientes.

F1:F6:F7:012 Normativa para regular los permisos e incentivos para los estudiantes deportistas que forman parte del Sistema Nacional de Educación.
D11:03: Diseño de un plan de incentivos, bonos que permitan mantener la fidelización de los clientes a través del tiempo y además el crecimiento de la demanda.

D4:O2 Ampliación de la infraestructura en cuanto a duchas, vestidores y casilleros para que el cliente tenga mayor comodidad en el servicio.

D2:04 Adquisición de nuevos implementos que permitan diferenciarse en el mercado y poder satisfacer las exceptivas de los deportistas D6:06 Establecer un cronograma de capacitación permanente para los instructores con la finalidad de actualizar sus conocimientos en las diferentes ramas deportivas y evitar en lo posible la deserción de los deportistas. 


\section{Cont... Cuadro 1}

\begin{tabular}{|c|c|c|}
\hline Amenazas & FA CONFRONTAR & DA EVITAR \\
\hline A1. Pérdida de imagen institucional & \multirow{6}{*}{$\begin{array}{l}\text { F3:F7:A5 Creación de un proce- } \\
\text { so médico que permita diseñar un } \\
\text { plan nutricional para los deportistas } \\
\text { siendo este un servicio adicional sin } \\
\text { costo que se preste a los deportis- } \\
\text { tas con el fin de obtener resultados } \\
\text { pronto en cuanto su rendimiento físi- } \\
\text { co y apariencia. } \\
\text { F7:A6 Diseño de un plan de se- } \\
\text { guimiento que permita, conocer la } \\
\text { situación psicológica del deportista. }\end{array}$} & \multirow{6}{*}{$\begin{array}{l}\text { D3A1 Contratación de un mayor número de } \\
\text { entrenadores capacitados y con experiencias, } \\
\text { que permita mejorar la imagen institucional de } \\
\text { la Federación Deportiva de Tungurahua. } \\
\text { D5:A3 Potencializar las estrategias de publici- } \\
\text { dad que permitan incrementar el mercado, dan- } \\
\text { do a conocer los servicios que brinda la FDT. }\end{array}$} \\
\hline $\begin{array}{l}\text { A2. Baja afluencia de deportistas en } \\
\text { deportes nuevos que oferta la FDT }\end{array}$ & & \\
\hline $\begin{array}{l}\text { A3. Presencia de otros centros que } \\
\text { fomentan la actividad física }\end{array}$ & & \\
\hline $\begin{array}{l}\text { A.4 Disminución del presupuesto asig- } \\
\text { nado por la Secretaria Nacional del } \\
\text { Deporte a la FDT }\end{array}$ & & \\
\hline $\begin{array}{l}\text { A5. Problemas nutricionales en los } \\
\text { deportistas }\end{array}$ & & \\
\hline $\begin{array}{l}\text { A6. Problemas extradeportivos de los } \\
\text { deportistas }\end{array}$ & & \\
\hline
\end{tabular}

Nota. Esta tabla muestra como resultado las estrategias obtenidas posterior al cruce realizado entre los factores internos (fortalezas y debilidades) y factores externos (oportunidades y amenazas).

Fuente: elaboración propia

Para continuar con el desarrollo se estructuró la matriz SAATY, jerarquizando las estrategias según el grado de importancia para la toma de decisiones del administrador institucional (tabla 3), a continuación, se las detalla:

a) Efectuar talleres y seminarios gratuitos promoviendo la realización del deporte y su afectación en la salud en las instituciones públicas y privadas que brinde apertura,

b) Implementar el pago del servicio mediante tarjetas de crédito, a fin de poder competir en el mercado ya que la competencia ya brinda este servicio y así poder llegar a un segmento más amplio de clientes,

c) Normativa para regular los permisos e incentivos para los estudiantes deportistas que forman parte del Sistema Nacional de Educación,

d) Diseño de un plan de incentivos, bonos que permitan mantener la fidelización de los deportistas a través del tiempo y además el crecimiento de la demanda, e) Ampliación de la infraestructura en cuanto a duchas, vestidores $y$ casilleros para que el deportista tenga mayor comodidad en el servicio,

f) Adquisición de nuevos implementos que permitan diferenciarse en el mercado y poder satisfacer las exceptivas de los deportistas,

g) Establecer un cronograma de capacitación permanente para los instructores con la finalidad de actualizar sus conocimientos en las diferentes ramas deportistas y evitar en lo posible la deserción de los deportistas,

h) Creación de un proceso médico que permita diseñar un plan nutricional para los deportistas, siendo este, un servicio adicional sin costo que se preste, con el fin de obtener resultados pronto en cuanto su rendimiento físico y apariencia,

i) Diseño de un plan de seguimiento que permita conocer la situación psicológica del deportista,

j) Contratación de un mayor número de entrenadores capacitados y 
Ríos Lara, Geovanny Fabricio; León Lara, Roxana Elizabeth;

Medina Guerrero, Wilson Eduardo y Romero Arévalo, Dennis Adolfo

Posicionamiento estratégico de la Federación Deportivade Tungurahua, Ecuador

con experiencia, que permita mejorar la imagen institucional de la federación,

k) Potencializar las estrategias de publicidad que permitan incrementar el mercado, dando a conocer los servicios que brinda la Federación Deportiva de Tungurahua.

\section{Tabla 3}

\section{Matriz SAATY}

\begin{tabular}{ccccccccccccccccccccccccccc} 
& A & B & C & D & E & F & G & H & I & J & K & a & b & c & d & e & f & g & h & i & j & k & Media & \%mportancia & Orden \\
\hline A & 1 & 3 & 9 & 1 & 5 & 7 & 1 & 9 & 9 & 3 & 1 & 0 & 0 & 0,2 & 0,1 & 0,2 & 0,2 & 0,1 & 0,3 & 0,3 & 0,2 & 0,1 & 0,15 & 14,94 & 2 \\
B & 5 & 1 & 9 & 5 & 5 & 1 & 3 & 9 & 7 & 1 & 1 & 0,1 & 0 & 0,2 & 0,4 & 0,2 & 0 & 0,3 & 0,3 & 0,2 & 0,1 & 0,1 & 0,16 & 16,39 & 1 \\
C & 9 & 9 & 1 & $1 / 5$ & $1 / 5$ & 1 & $1 / 5$ & 1 & 1 & $1 / 5$ & $1 / 5$ & 0,2 & 0,1 & 0 & 0 & 0 & 0 & 0 & 0 & 0 & 0 & 0 & 0,04 & 4,2 & 11 \\
D & 1 & 5 & 9 & 1 & 5 & 7 & 1 & 1 & 1 & 1 & 1 & 0 & 0,1 & 0,2 & 0,1 & 0,2 & 0,2 & 0,1 & 0 & 0 & 0,1 & 0,1 & 0,09 & 9,38 & 5 \\
E & 3 & 5 & 1 & $1 / 5$ & 1 & 9 & $1 / 5$ & 1 & 3 & $1 / 5$ & 1 & 0,1 & 0,1 & 0 & 0 & 0 & 0,3 & 0 & 0 & 0,1 & 0 & 0,1 & 0,06 & 6,29 & 8 \\
F & 5 & 7 & 9 & 3 & 1 & 1 & $1 / 5$ & 1 & 1 & 1 & 3 & 0,1 & 0,1 & 0,2 & 0,2 & 0 & 0 & 0 & 0 & 0 & 0,1 & 0,2 & 0,09 & 8,85 & 6 \\
G & 5 & 7 & 9 & $1 / 5$ & 1 & 3 & 1 & $1 / 5$ & 5 & 1 & 5 & 0,1 & 0,1 & 0,2 & 0 & 0 & 0,1 & 0,1 & 0 & 0,1 & 0,1 & 0,3 & 0,1 & 10,11 & 4 \\
H & 9 & 9 & 1 & $1 / 5$ & $1 / 9$ & $1 / 5$ & $1 / 5$ & 1 & 1 & 1 & 1 & 0,2 & 0,1 & 0 & 0 & 0 & 0 & 0 & 0 & 0 & 0,1 & 0,1 & 0,05 & 4,86 & 9 \\
I & 9 & 9 & 1 & $1 / 5$ & $1 / 9$ & $1 / 5$ & $1 / 5$ & 1 & 1 & 1 & 1 & 0,2 & 0,1 & 0 & 0 & 0 & 0 & 0 & 0 & 0 & 0,1 & 0,1 & 0,05 & 4,86 & 10 \\
J & 5 & 7 & 9 & 1 & 1 & 1 & 1 & 1 & 5 & 1 & 1 & 0,1 & 0,1 & 0,2 & 0,1 & 0 & 0 & 0,1 & 0 & 0,1 & 0,1 & 0,1 & 0,08 & $\mathbf{8 , 0 4}$ & 7 \\
K & 7 & 7 & 1 & 1 & 3 & 3 & 3 & 3 & 1 & 5 & 1 & 0,1 & 0,1 & 0 & 0,1 & 0,1 & 0,1 & 0,3 & 0,1 & 0 & 0,3 & 0,1 & 0,12 & 12,07 & 3 \\
\hline Totales & 59 & 69 & 59 & 13,1 & 22,4 & 33 & 11 & 28 & 35 & 15,5 & 16 & 1 & 1 & 1 & 1 & 1 & 1 & 1 & 1 & 1 & 1 & 1 & & 100 & \\
\hline
\end{tabular}

Nota: La tabla describe la puntuación dada a cada una de las estrategias diseñadas de acuerdo a los siguientes parámetros: 1 = Igual de Importante; 3 = Ligeramente más importante (1/3 Ligeramente menos importante); 5 = Más importante (1/5 Menos importante); $7=$ Bastante importante $(1 / 7$ Bastante menos importante); 9 = Mucho más importante (1/9 Mucho menos importante).

Fuente: elaboración propia

Con base a la tabla 3, se seleccionó a las estrategias según su valoración destacando las siguientes según su grado de importancia:

1. La estrategia C $(33,40 \%)$. Normativa para regular los permisos e incentivos para los estudiantes deportistas que forman parte del Sistema Nacional de Educación.

2. La estrategia J (11,86\%). Contratación de un mayor número de entrenadores capacitados y con experiencias, que permita mejorar la imagen institucional de la Federación Deportiva de Tungurahua.

3. La estrategia I $(10,01 \%)$. Diseño de un plan de seguimiento que permita conocer la situación psicológica del deportista.

4. La estrategia $H(9,74 \%)$. Creación de un proceso médico que permita diseñar un plan nutricional para los deportistas siendo este un servicio adicional sin costo que se preste, con el fin de obtener resultados pronto en cuanto su rendimiento físico y apariencia.

5. La estrategia K $(9,04 \%)$. Potencializar las estrategias de publicidad que permitan incrementar el mercado, dando a conocer los servicios que brinda la Federación 
Deportiva de Tungurahua.

6. La estrategia E (7,99\%). Ampliación de la infraestructura en cuanto a duchas, vestidores y casilleros para que el deportista tenga mayor comodidad en el servicio.

7. La estrategia $F(6,19 \%)$. Adquisición de nuevos implementos que permitan diferenciarse en el mercado y poder satisfacer las exceptivas de los deportistas.

8. La estrategia D (4,09\%). Diseño de un plan de incentivos, bonos que permitan mantener la fidelización de los clientes a través del tiempo y además el crecimiento de la demanda.

9. La estrategia A (3,65\%). Efectuar talleres y seminarios gratuitos promoviendo la realización del deporte y su afectación en la salud en las instituciones públicas y privadas que brinde apretura.

10. La estrategia $G(2,46 \%)$. Establecer un cronograma de capacitación permanente para los instructores con la finalidad de actualizar sus conocimientos en las diferentes ramas deportistas y evitar en lo posible la deserción.

11. La estrategia B $(1,56 \%)$ Implementar el pago del servicio mediante tarjetas de crédito, a fin de poder competir en el mercado, dado que, la competencia en la actualidad ya brinda este servicio y así poder llegar a un segmento más amplio de clientes.

Finalmente, en la fase 3 , se estableció el plan de acción y control de las estrategias diseñadas para el posicionamiento estratégico de la Federación Deportiva de Tungurahua. En este sentido, según el plan de acción determinado, la institución debe presentar su organización como una unidad eficaz en el campo estableciendo unidades de comunicación con la industria e implementando las estrategias adecuadas que permitan mantenerse vigentes en el mercado sobre todo aquellas en las cuales intervenga directamente las Tecnologías de la Información y Comunicación (TIC) (Lalaleo, Bonilla y Robles, 2021).

\section{Hallazgos en contexto}

En el ámbito empresarial una de las mayores preocupaciones de las distintas organizaciones de cualquier sector ha sido el tema de la gestión estratégica, convirtiéndose la misma en un factor de fracaso en muchas instituciones (Gajda, Mikalauskas \& Navickas, 2020). En este sentido, expertos en ciencias de la gestión, especialmente del área estratégica, introdujeron la planificación estratégica con la finalidad de que las organizaciones puedan transformar las estrategias de un escenario subjetivo como lo llevaban hasta hace unos años atrás a un escenario operativo en donde se puedan cumplir metas y objetivos que permitan responder positivamente a una organización ante eventos que pudieran generarse.

Autores como Andrade (2016) e Hidalgo et al. (2016), señalan que el plan estratégico de marketing debe estar orientado a los lineamientos del diseño de estrategias de posicionamiento de la organización, no obstante, resulta necesario destacar que no es una fórmula universal para todas las empresas pues depende de los factores y rasgos que definen el negocio al que se aplicará la planificación.

En este contexto, de acuerdo al estudio realizado, la Federación Deportiva de Tungurahua presenta una serie de inconvenientes asociados 
al ámbito de la gestión estratégica que limitan su desarrollo y su posicionamiento, muestra de ello son los resultados encontrados, pues los mismos muestran en el contexto interno una prevalencia de las debilidades por sobre las fortalezas demostrando de esta manera que la institución internamente es débil, mientras que, en el contexto externo, las amenazas fueron más que las oportunidades que ofrece el mercado, encontrándose la unidad de estudio en una industria que afronta una serie de dificultades.

Con la finalidad de contrarrestar este panorama y proyectar un crecimiento institucional a través de un posicionamiento en el mercado, posterior al diagnóstico realizado a través de las matrices FODA, MEFI y MEFE se diseñaron estrategias en función de las necesidades de la Federación Deportiva de Tungurahua. Esta metodología aplicada es similar al presentado en un estudio desarrollado por Nazari y Pour (2013) quienes a través de los principios teóricos del modelo de planificación estratégica propuesto por David (2003), se han encargado de diseñar una serie de estrategias mediante las herramientas de gestión modernas (FODA, MEFI, MEFE), dichas estrategias fueron direccionadas hacia la presentación de nuevos servicios y una mayor difusión de la Federación Nacional de Sepaktakraw de Singapur con la finalidad de posicionarse en el mercado deportivo de aquel país.

En este mismo orden de ideas, se cita el trabajo desarrollado por Peñafiel et al. (2020) quien pone de manifiesto que para la formulación de la estrategia se hace necesario elaborar un diagnóstico tanto del entorno interno como externo de la institución, para ello, se deben valorar sus fortalezas y sus debilidades y mirar en el contexto externo las oportunidades y amenazas. Para el proceso de formulación de estrategias es importante que se analicen un conjunto de matrices (FODA, MEFI, MEFE) las cuales son concatenadas para establecer acciones que permitan aprovechar las oportunidades, capitalizar las fortalezas, minimizar las debilidades y por su puesto tratar en lo posible de evitar que las amenazas tengan un impacto significativo sobre el desempeño de la institución.

\section{Conclusiones}

La administración estratégica es una parte integral de la administración exitosa del día a día. La gestión estratégica es un proceso continuo, dinámico y coherente que permite a una organización adaptarse a los cambios externos y utilizar eficazmente sus recursos y su potencial. Generalmente, la gestión estratégica consta de tres etapas que se encuentran interrelacionadas de diversas formas, es decir, análisis estratégico, desarrollo e implementación de estrategias.

Al diseñar el plan estratégico de marketing se consideró las estrategias de posicionamiento de acuerdo al valor agregado que la institución debe entregar a los deportistas (consumidores) como: entrenadores con experiencia, reconocimiento y pertinencia sobre la marca (Federación Deportiva de Tungurahua), infraestructura con equipos idóneos para las actividades, promociones, bonos e incentivos que motiven al deportista a formar parte de la institución.

Para generar una mayor profundidad al estudio, se apertura una futura línea de investigación, en la cual se deba analizar el factor humano 
y su incidencia en la ejecución de la planificación de la organización, ya que, entregarían una apreciación más clara sobre los problemas de ejecución y cumplimiento de las metas de la institución.

\section{Referencias Bibliográficas}

Abdullah, N. (2015). How does marketing strategy influence firm performance Implementation of marketing strategy for firm success. International Journal of Innovation and Economic Development, 1(3), 7-15. https://doi.org/10.18775/iii ed.1849-7551-7020.2015.13.2001

Andrade, D. (2016). Estrategias de marketing digital en la promoción de marca ciudad. Revista Escuela de Administración de Negocios, (80), 59-72. https://www.redalyc.org/ pdf/206/20645903005.pdf

Bonilla, D., Delgado, N., y Fajardo, G. (2020). Branding, un elemento necesario del marketing estratégico en la Cámara de Comercio de Ambato. Revista Eruditus, 1(2), 9-26. $\quad$ https://doi.org/10.35290/ re.v1n2.2020.278

Bonilla, D., Salinas, D., Lalaleo, F., y Velastegui, S. (2018). Marketing estratégico en redes sociales de las organizaciones. Un abordaje $360^{\circ}$. Ciencia y Tecnología, 1(16), 1-19. https://doi.org/10.47189/rcct. v18i17.154

Carpio, A., Hancco, M. S., Cutipa, A. M., y Floresi, E. (2019). Estrategias del marketing viral y el posicionamiento de marca en los restaurantes turísticos de la Región de Puno. Comuni@cción: Revista de Investigación en Comunicación y Desarrollo, 10(1), 70-80. https://doi. org/10.33595/2226-1478.10.1.331
Chang, J., \& Tun-Min, C. (2015). Is fast fashion sustainable? The effect of positioning strategies on consumers attitudes and purchase intentions. Social Responsibility Journal, 11(4), 853-867. https://doi.org/10.1108/ SRJ-07-2014-0095

Copulsky, J. R., \& Wolf, M. J. (1990). Relationship Marketing: Positioning for the Future. Journal of Business Strategy, 11(4), 16-20. https://doi. org/10.1108/eb060069

David, F. (2003). Conceptos de Administración Estrátegica. (9na. ed.). Pearson Educación.

Franco, J., Restrepo, J., y Sánchez, J. (2014). La gestión del mercadeo: un aporte a la competitividad de las pequeñas empresas del sector servicios en Medellín. Pensamiento y Gestión, 37, 150174. http://www.redalyc.org/articulo. oa? id $=64632634008$

Fuchs, C., \& Diamantopoulos, A. (2010). Evaluating the effectiveness of brand-positioning strategies from a consumer perspective. European Journal of Marketing, 44(11/12), 1763-1786. https://doi. org/10.1108/03090561011079873

Gajda, W., Mikalauskas, R., \& Navickas, V. (2020). Employees assessment of strategic management opportunities in Sport Federation. Journal of Interdisciplinary Research $A D$ Alta, 188-194.

Hernández, R., Fernández, C., y Baptista, P. (2014). Metodología de la investigación. (6ta. ed.). Mc Graw Hill.

Herrera, J. (2017). Guía metodológica para la elaboración del plan de marketing deportivo. 3C Empresas, 6(1), 1-21. https://ojs.3ciencias. com/index.php/3c-empresa/article/ view/408 
Ríos Lara, Geovanny Fabricio; León Lara, Roxana Elizabeth;

Medina Guerrero, Wilson Eduardo y Romero Arévalo, Dennis Adolfo

Posicionamiento estratégico de la Federación Deportivade Tungurahua, Ecuador

Hidalgo, P., Rodríguez, J., y Carmenate, R. (2020). Aplicación de la dirección estratégica en la gestión del deporte en Granma (Original). Revista científica Olimpia, 13(41), 72-84. https://revistas.udg.co.cu/index.php/ olimpia/article/view/1293

Hume, C., \& Hume, M. (2015). The Critical Role of Internal Marketing in Knowledge Management in Notfor-Profit Organizations. Journal of Nonprofit \& Public Sector Marketing, 27(1), 37-41. https://doi.org/10.1080/ 10495142.2014.934567

Jeanpert, S., \& Paché, G. (2016). Successful multi-channel strategy: mixing marketing and logistical issues. Journal of Business Strategy, 37(2), 12-19. https://doi.org/10.1108/ JBS-05-2015-0053

Kitchen, P., \& Burgmann, I. (2015). Integrated marketing communication : making it work at a strategic level. Journal of Business Strategy, 36(4), 34-39. https://doi.org/10.1108/JBS05-2014-0052

Lalaleo, F., Bonilla, D., y Robles, R. (2021). Tecnologías de la Información y Comunicación exclusivo para el comportamiento del consumidor desde una perspectiva teórica. Retos Revista de Ciencias de la Administración y Economía, 11(21), 147-164. https://doi.org/10.17163/ ret.n21.2021.09

López, O., Beltrán, C., Morales, R., y Cavero, O. (2018). Estrategias de marketing digital por medio de redes sociales en el contexto de las PYMES del Ecuador. Revista CienciAmérica, $7(2), \quad 39$. https://doi.org/10.33210/ ca.v7i2.167

Maritz, R., \& Du Toit, A. (2018). The practice turn within strategy: Competitive intelligence as integrating practice. South African Journal of Economic and Management
Sciences, 21(1), 1-14. https://doi. org/10.4102/sajems.v21i1.2059

Mourad, M. (2010). Internationalisation: a new positioning strategy in the higher education market. Int. J. Management in Education, 4(2), 185-199. https://doi.org/10.1504/ IJMIE.2010.030875

Nazari, R. \& Pour, H. (2013). Development of perspectives and strategic planning in Sport. Management and administrative sciences review, 2(2), 104-113.

Nogales, J. (2006). Estrategias de marketing en clubes deportivos. Revista de Ciencias del Deporte, 2(3), 45-52. https://www.redalyc.org/ pdf/865/86502301.pdf

Panamá, C., Erazo, J., Narváez, C., y Mena, S. (2019). El Marketing como estrategia de posicionamiento en empresas de servicios. Revista Cientifica Dominio de Las Ciencias, $5(3), \quad 784-802 . \quad$ http://dx.doi. org/10.23857/dc.v5i3.988

Peñafiel, G., Acurio, J., Manosalvas, L., y Burbano, B. (2020). Formulación de estrategias para el desarrollo empresarial de la constructora Emanuel en el cantón La Maná. Revista Universidad y SOciedad, 12(4), 45-55.

Rajiv, S., Dutta, S., \& Dhar, S. K. (2002). Asymmetric Store Positioning and Promotional Advertising Strategies: Theory and Evidence. Marketing Sciences, 21(1), 74-96. https://doi. org/10.1287/mksc.21.1.74.160

Rasooli, S., Bkhsheshi, M., \& Zareian, $\mathrm{H}$. (2018). Brand positioning of the Sport Sciences Research Institution of Iran using Perceptual Mapping Technique. Annals of Applied Sport Science, 6(1), 103-113. https://doi. org/10.29252/aassjournal.6.1.103 
Romero, D., y Moreno, K. (2018). Estrategias para el posicionamiento de mercado en la Federación Deportiva de Tungurahua. [Tesis de posgrado, Pontificia Universidad Católica del Ecuador]. https:// repositorio.pucesa.edu.ec/ handle/123456789/2542

Ruiz, D., Bonilla, D,. y Masaquiza, C. (2018). Ajuste estratégico en la cadena de suministros para la creación de valor de la marca Jean Up. Universidad y Sociedad, Revista Científica de La Universidad de Cienfuegos, 10(1), 25-32. https://bit. Iy/3xP1sKo

Suárez-Amaya, W., Díaz-Barrios, J. y Pereira, M. (2021). Perfil competitivo como herramienta para la gestión estratégica de la investigación en universidades. Hallazgos 18 (35), 259-286. https://doi. org/10.15332/2422409X.5768

\begin{tabular}{ccc} 
Vidaller, L. (2014). & \multicolumn{2}{c}{ Estrategias } \\
empresariales & \multicolumn{2}{c}{ competitivas } \\
en el mercado & de las \\
telecomunicaciones. & [Tesis de
\end{tabular}

posgrado, Universidad Pontificia Comillas]. https://repositorio. comillas.edu/xmlui/bitstream/ handle/11531/395/TFG000260. pdf?sequence $=1$ \&isAllowed $=y$

Villalón, J., Hidalgo, P., Castellanos, I., y García, P. (2017). La utilización de matrices estratégicas en la dirección de equipos de baloncesto. Olimpia Revista de la Facultad de Cultura Fisica de la Universidad de Granma, 14(43), 206-220.

Wijaya, H., \& Sirine, H. (2016). Strategi segmenting , targeting , positioning serta strategi harga pada perusahaan kecap blekok di cilacap. AJIE Asian Journal of Innovation and Entrepreneurship, 1(3), 175-190. https://journal.uii.ac.id/ajie/article/ view/6996/6150

Zaheer, A., \& Rashid, A. (2017). Analyzing the role of public sector marketing in improving social effectiveness: a case study from Anhui province, China. International Review on Public and Nonprofit Marketing, 14(1), 57-71. https://doi.org/10.1007/s12208-016$\underline{0162-y}$ 